\title{
Education about safety for patients with diabetes who exercise is essential to encourage lifestyle modification
}

\section{Editorial}

Patients with diabetes are encouraged to exercise regularly, yet there is a gap with regard to how to exercise safely. A program was created to provide a seminar for patients and healthcare professionals to educate about the risks and benefits of exercise and to provide information about proper monitoring parameters. The seminar provides insights about many risks that patients have while doing an exercise especially an intensive aerobic exercise. The discussion includes what foods can be consumed before and after exercise, valuable monitoring parameters, and problems patients may encounter as they exercise. The significance of this project is that it is one of very few programs that targets building confidence in exercise for patients, patients' support network, and providers in the community. In addition, the seminar also targets healthcare professionals through power-point presentations and installs confidence in providers by providing them with information to discuss with their patients. Both the seminar for patients and the presentation for healthcare professionals include an evaluation of knowledge learned and impact on reduction of potential adverse effects for patients during and after they engage in exercise.

As a healthcare professional and a diabetes educator I often recommend lifestyle modification to my patients and I follow recommendations given by organization such as the American Diabetes Association (ADA). ADA recommends regular scheduled Exercise duration for individuals with diabetes. Recommendations include exercising 150minutes per week of moderate intensity exercise. In addition, patients are also advised to consider resistance training for 2days or more per week. Exercise is advised by the ADA at least 3 days/week but no more than 2 days with no exercise. ${ }^{1}$ The goal of exercise in patients with diabetes is weight loss. The benefits of weight loss are improvement of diabetes, lipid profile and reduction in blood pressure. ${ }^{2}$ When I recommend exercise, I am often met with resistance and many excuses. But, I have a growing number of patients that embrace the idea. Many patients that consider an exercise routine are at risk for hypoglycemia not only from antidiabetic medications but also from other medications such as Beta blockers for example. ${ }^{3}$ Yet, very few of these patients are aware of this risk for hypoglycemia, and even fewer patients know what to do when they experience hypoglycemia. When adverse effects occur during or after exercise, I find that many individuals simply quit and loose the interest to engage in any exercise. The ADA and other organizations do not provide enough information to ensure safety during and after exercise.

The seminar, which is provided by a board certified diabetes educator, is created to fill the gap. The program is divided into 5 one hour sessions that are provided in a dojo in central New Jersey that has a program for weight loss through an intensive 45 minute aerobic exercise program. Participants include patients with diabetes or pre-diabetes with or without diabetes complications, including their network support of family and friends. Each seminar addresses different topics including risk and benefits of exercise, nutrition prior
Volume 2 Issue 3 - 2015

\author{
Samuel S Grossman \\ Hunter College of City University of New York, USA
}

Correspondence: Samuel S Grossman, Adjunct Associate Professor, Hunter College of the City University of New York, 695 Park Ave, New York, USA, Tel 2 I 248| 4465,

Email sgrossm@hunter.cuny.edu

Received: June 16,2015 | Published: June 19, 2015

and after exercise, signs and symptoms of hypoglycemia, importance of monitoring (finger sticks, blood pressure, feet, change in weight, hemoglobin A1c, lipid profile, kidney and liver function, pulmonary function test for patients at risk), problem solving skills and sick day management. The seminar is an instruction and discussion based presentation. Patients receive a handout and printed information is discussed. Participants engage in a discussion after the instructional component and fill a pre-test and a post-test. The discussion covers barriers and challenges that participants face in their daily life. The program also includes a separate, power point, 1hour, case-based presentation targeting healthcare professionals from different disciplines. The presentation intends to educate healthcare professionals about the curriculum of the program to the patients and elicit questions to clarify misunderstanding about safety with exercise. Since the inception of the program, I am already seeing significant improvement in the confidence of the participants and in the show rate to seminars. Many participants comment that they wished they learned this information from their providers. I believe that moving forward with this program will prove that patient education as included in this curriculum can empower patients to engage in physical activity and maintain an exercise schedule with reduction of adverse effect. Moreover, I predict that once the program spreads to the healthcare professional sector, providers will be more willing to embrace lifestyle modification, including exercise and will promote it to their patient population. It is my hope that this educational program will provide inspiration to the creation of many such programs across the U.S and around the world.

\section{Acknowledgements}

The seminar is provided through a grant from Quest Diagnostic Laboratories.

\section{Conflict of interest}

Author declares that there is no conflict of interest.

\section{References}

1. Professional Practice Committee. Standard of medical care in diabetes-2015. Diabetes care. 2015;38(Suppl 1):S1-S91. 
2. Ribeiro F, Costa R, Mesquita-Bastos J. Exercise training in the management of patients with resistant hypertension. World J Cardiol. 2015;7(2):47-51.
3. Medline Plus. Drug-induced hypoglycemia. 2015. 